\title{
Interspecies systems biology (a.k.a. interspecies genetics)
}

The power of genetics in the worm, and in the bacteria that worms eat, is harnessed for a systems view of the effect of diet on a host organism.

"Tell me what you eat, and I will tell you what you are," the gastronome Brillat-Savarin wrote. To put it differently: the complex network of relationships between an organism, its metabolism, its diet and its gut microbiota is important for the state of the host-healthy or diseased, well nourished or not, fat or thin. These relationships are most commonly studied in humans and in the mammalian rodent models that (it is hoped) mimic them. But lately, evidence indicates that the humble nematode Caenorhabditis elegans can contribute, too.

In a recent paper, Marian Walhout and colleagues at the University of Massachusetts Medical School, Worcester, identify vitamin $B_{12}$ as a dietary factor that can affect both gene expression programs and the rate of development in the worm (Watson et al., 2014). In earlier work, Walhout and colleagues had observed that the species of bacterium fed to worms has measurable effects on worm lifespan, developmental rate and egg laying. Worms that ate their typical laboratory diet of Escherichia coli (an enteric bacterium that a worm is unlikely to encounter at high concentration in its natural habitats) developed more slowly, but lived longer and laid more eggs, than worms fed the bacterium Comamonas aquatica.

The researchers also identified changes in worm gene expression that depended on diet. On this basis, they developed a fluorescent reporter worm strain that could be used to screen for genes involved in the response. On an E. coli diet, the reporter strain (which carries GFP driven by the promoter of the acyl-CoA dehydrogenaseencoding gene $a c d h-1$ ) has bright green fluorescence in the cells of its intestine. On a Comamonas diet, the reporter is not expressed, and the worm is dark. The researchers determined in mixing experiments that Comamonas most probably secretes a factor that represses this reporter in the worm. Previously, Walhout and colleagues used the reporter strain to screen for worm genes that, when mutant, could repress or enhance the response to diet.
The idea they hit upon in their new work was to use an interspecies approach: they screened mutant libraries of the dietary bacteria, looking for bacterial genes that when mutated affect normal reporter expression patterns. They screened the E. coli deletion collection and identified 77 mutants, many of them in metabolic genes, that affected reporter expression. For Comamonas, they did a transposon mutagenesis screen and identified five mutants with ectopic reporter expression.

Combining the results from the interspecies genetics experiments with those of a secondary screen in which they added a panel of nutrients and metabolites to reporter worms, Walhout and colleagues honed in on vitamin $B_{12}$ as a key player. Sure enough, Comamonas makes more vitamin $\mathrm{B}_{12}$ than E. coli, and giving worms a vitamin supplement on the E. coli diet suppressed the dietary reporter and affected some (though not all) of the developmental traits. Specifically, worm lifespan was not affected by adding vitamin $\mathrm{B}_{12}$ to an E. coli diet, but the animals developed faster and laid fewer eggs.

Using interspecies genetics to study hostgut microbiota relationships would be difficult or impossible in mammalian hosts because of increased host and diet complexity. The worm, furthermore, may be amenable to studies with more complex dietary mixtures or those derived from natural food sources. The transparency of the animal and the large size of its intestinal cells could even make it possible to add higher-resolution imaging approaches to the already tremendous power of worm and bacterial genetics. Notably, many of the genes identified in the worm as playing a role in sensing bacterial nutrients have orthologs implicated in human metabolic disease. The worm may have been developed primarily as a model for development and neurobiology, but there is reason to think it could also tell us something about how our diet makes us what we are. Natalie de Souza

RESEARCH PAPERS

Watson, E. et al. Interspecies systems biology uncovers metabolites affecting $C$. elegans gene expression and life history traits. Cell 156, 759-770 (2014). 